\title{
THE INFLUENCE OF NON-AXISYMMETRIC PULSE SHAPER POSITION ON SHPB EXPERIMENTAL DATA
}

\author{
RoBert PANOWICZ \\ Military University of Technology, Faculty of Mechanical Engineering, Warsaw, Poland \\ e-mail: robert.panowicz@wat.edu.pl \\ JaCEK Janiszewski, KrZyszTof Kochanowski \\ Military University of Technology, Faculty of Mechatronics and Aviation, Warsaw, Poland
}

\begin{abstract}
The results of experimental and numerical analysis of the influence of the non-axisymmetric pulse shaper position on recorded wave signals in the split Hopkinson pressure bar experiment are presented. The paper focuses attention on the problem of wave signal disturbances caused by a bending wave resulting from non-axisymmetric pulse shaper positions and, moreover, different shaper thickness, striker impact velocities and Wheatstone bridge configurations. The obtained results of analyses indicate that the effect of the non-axisymmetric pulse shaper position may be neglected if deviation from the bar axis does not exceed $20 \%$.
\end{abstract}

Keywords: split Hopkinson pressure bar, pulse shaping technology, high-strain-rate testing

\section{Introduction}

The most popular experimental arrangement for investigating dynamic behaviour of materials at high strain rates within the range $10^{2}$ to $5 \cdot 10^{4} \mathrm{~s}^{-1}$ is the split Hopkinson pressure bar (SHPB), also known as Kolsky bar. The original setup of SHPB in compression was constructed by Herbert Kolsky, who developed in 1949 also the theoretical basis of the method (Kolsky, 1949). In general, the SHPB technique has been widely used to determine dynamic properties of a variety of engineering materials such as metals and their alloys, ceramics, polymers and elastomers, composites, shape memory alloys, foams, and even biological tissues (Chen and Song, 2011).

The SHPB setup usually consists of the striker bar and two long bars called the input and output bars with the same diameter and material (typically high strength material like maraging steel). The material sample is placed between the bars, and it is loaded by a stress impulse generated by the striker impact applied to the front surface of the input bar. This generates a trapezoidal stress impulse (incident wave) which travels through the impacted bar. When the elastic wave reaches the specimen, due to mismatch of mechanical impedances between the bar and the specimen material, a part of the incident wave is reflected back (reflected wave) and the rest of the incident wave is transmitted through the specimen. It compresses the specimen with high rates, and rest of the wave travels to the output bar as a transmitted wave. The incident and reflected signals are recorded by strain gages which are glued on the input bar, whereas the transmitted signals are sensed by strain gages located on the output bar.

In split Hopkinson pressure bar (SHPB) experiments, besides specimen geometry, the profile of the incident pulse is the only controllable parameter to subject the specimen to the desired testing conditions. The shape of the incident stress wave is usually controlled through pulse techniques (Chen and Song, 2011). There are different techniques of incident pulse shaping, such as modification of the striker geometry (Bekker et al., 2015; Cloete et al., 2009), using a preloading bar (Ellwod et al., 1982; Foley et al., 2010) or by using a pulse shaper method (Chen 
and Luo 2004; Lu and Li, 2010; Chen and Song, 2011), where a small disc made of a ductile material is glued with grease on the impact end of the input bar (Fig. 1). This technique is mainly used for facilitating stress equilibrium and constant strain rate deformation in the specimen (Vecchio and Jiang, 2007). Moreover, the dispersion of incident and reflected waves propagated in SHPB bars can be physically minimized through a pulse shaper, which plays role of a mechanical filter to damp undesired high-frequency signal components in the incident pulse (Chen and Song, 2011).

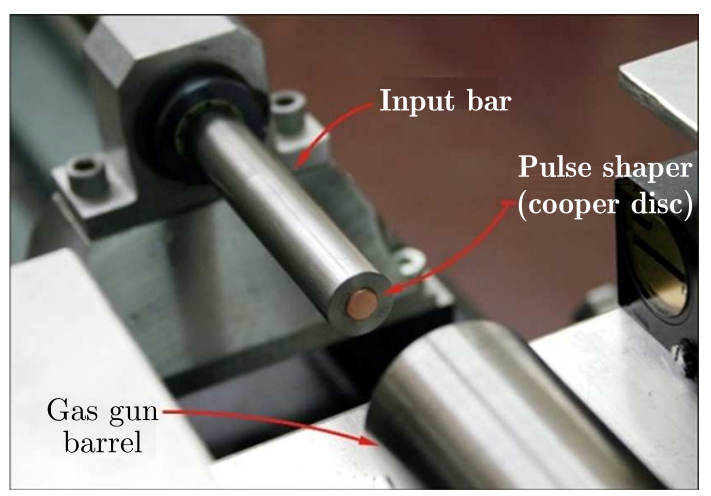

Fig. 1. View of a pulse shaper placed on the impact face of the input bar

Nowadays, the pulse shaper technique is a well-known and commonly used method. The experimental studies have been carried out using pulse shapers not only for metal specimens but also for materials such as rubber (Song and Chen, 2005), plexiglass (Naik and Yernamma, 2008), fabric (Hsiao and Daniel, 1998) and ceramic (Chen and Luo, 2004).

One of the first works devoted to this technique were published by Franz and Follansbee (1984) and Follansbee (1985). They investigated the influence of pulse shaper thicknesses ranging from 0.1 to $2.0 \mathrm{~mm}$ on shaping the incident pulse, minimization of dispersion effects and dynamic stress equilibrium in the sample. More recently, the pulse shaper technique was widely considered by Chen et al. (2003). They used a layered pulse shaper consisting of two disks made of copper/mild steel to investigate small-strain behaviour of mild steel, i.e., through a material dynamic test in the initial stage of both elastic and plastic deformation. The above-mentioned authors proposed also an original use of the pulse shaper technique to determine dynamic stressstrain loops for engineering materials (Song and Chen, 2004). They used an arrangement of two pulse shapers placed in front of and behind the flange for precise control of loading and unloading a portion of the incident pulse. Thanks to this, it was possible to obtain a similar rise and fall loading time of the stress wave.

Great contribution to the development of the pulse shaping technique was brought by a team headed by Vecchio. For example, Vecchio and Jiang (2007) proposed making use of a pulse shaper made of a high strength, high-work-hardening rate material to obtain a half sinusoidal incident pulse.

The issue of the pulse shaper in the SHPB was based on analytical and numerical considerations. The analytical model of the influence of a deformable pulse shaper made of OFHC copper on the incident pulse was developed by Nemat-Nasser et al. (1991). The analytical model was also developed by Frew et al. $(2002,2005)$ for homogeneous and layered pulse shapers made of different materials. In turn, Ramirez and Rubio-Gonzalez (2006) used the finite element method to study the effect of incident pulse rise time and the selection of materials and geometric parameters of the pulse shaper on wave dispersion. In their research, they used shapers made of copper, 7039 aluminum and 4340 steel. Application of the copper pulse shaper allowed alleviating the wave dispersion. In turn, the influence of diameter and thickness of a pulse shaper made of C10200 copper as a function of the striker velocity was investigated by Naghdabadi et al. 
(2012) using numerical analysis and experimental research. On the basis of the research results, they formulated general guidelines to properly design a pulse shaper.

A proper choice of the pulse shaper geometry and material is particularly important in the case of brittle materials for which the value of failure strain is less than $1 \%$. In this case, special attention should be paid to minimization of the wave dispersion, quick receiving of dynamic stress equilibrium and nearly constant strain rates over most of the test duration. Therefore, the technique of shaping the incident pulse needs to guarantee the possibility of receiving a stress wave of a suitable shape. Ellwood et al. (1982), Li and Xua (2009), and Shemirani et al. (2016) demonstrated that a half sinusoidal incident pulse is suitable for testing brittle materials.

Despite the development of the analytical model and many numerical models concerning the influence of the pulse shaper geometry on the incident pulse, the problem of selection of a pulse shaper for testing different materials is usually solved via try and error approach.

The basic requirement for the desired application of a pulse shaper is to place the pulse shaper on the front face of the input bar axisymmetrically. However, in routine experimental practice, no special attention is paid to the pulse shaper positioning and, it is usually carried out more or less carefully. It may be supposed that the non-axisymmetrical fixing of the pulse shaper will cause additional disturbances of measured signals arising from formation and propagation of bending waves in the bars. These disturbances will occur especially when the measurement system is based on a Wheatstone bridge arrangement as a quarter- or half-bridge circuit. The half-bridge is commonly used in the SHPB experimental technique, in which bars with small diameter $(3-6 \mathrm{~mm})$ are used. In these cases, problems with gluing strain gauges required to obtain a full Wheatstone bridge occur. It can be also supposed that the disturbances caused by the non-axisymmetrical position of the pulse shaper will be significant for bars with low stiffness (e.g. plexiglas). It appears that the errors resulting from these conditions will be mainly expressed in the incorrect determination of the strain value in the sample. Therefore, in this paper, experimental investigation and numerical analysis are undertaken to estimate the effect of the pulse shapers non-axisymmetric position on stress waves in SHPB and the material dynamic response.

The influence of different pulse shaper positions and different shaper thickness for a quarterand a half-Wheatstone bridge configuration and three impact velocities of $10,12.5$ and $15 \mathrm{~m} / \mathrm{s}$ are subjected to considerations.

The paper is organized as follows: Section 2 is dedicated to the methodology of numerical and experimental investigations. Numerical and experimental results are collected in Section 3, where the influence of different pulse shapers positions and different thickness of the shapers are analyzed. Moreover, the influence of striker velocity on the pulse propagation and the sample dynamic response depending on the non-axisymmetric pulse shaper position is also considered. A summary and conclusions are presented in the final Section.

\section{Methodology of experimental and numerical investigations}

\subsection{Experimental setup}

Numerical analysis of the main problem of the present paper has been verified experimentally with the use of a split Hopkinson pressure bar stand presented in Fig. 2. It mainly consists of a launching system (air pressure gun), striker bar, input bar, output bar (bar system), velocity measuring device and a computer-controlled high-frequency data acquisition system.

The input bar and the output bar are $1200 \mathrm{~mm}$ long, while the striker length is $250 \mathrm{~mm}$. Both the bars and the striker have a common diameter of $12.05 \mathrm{~mm}$ and are made of maraging steel of grade MS350 (nominal quasi-static yield strength $R_{0.2}=2300 \mathrm{MPa}$, sound speed 


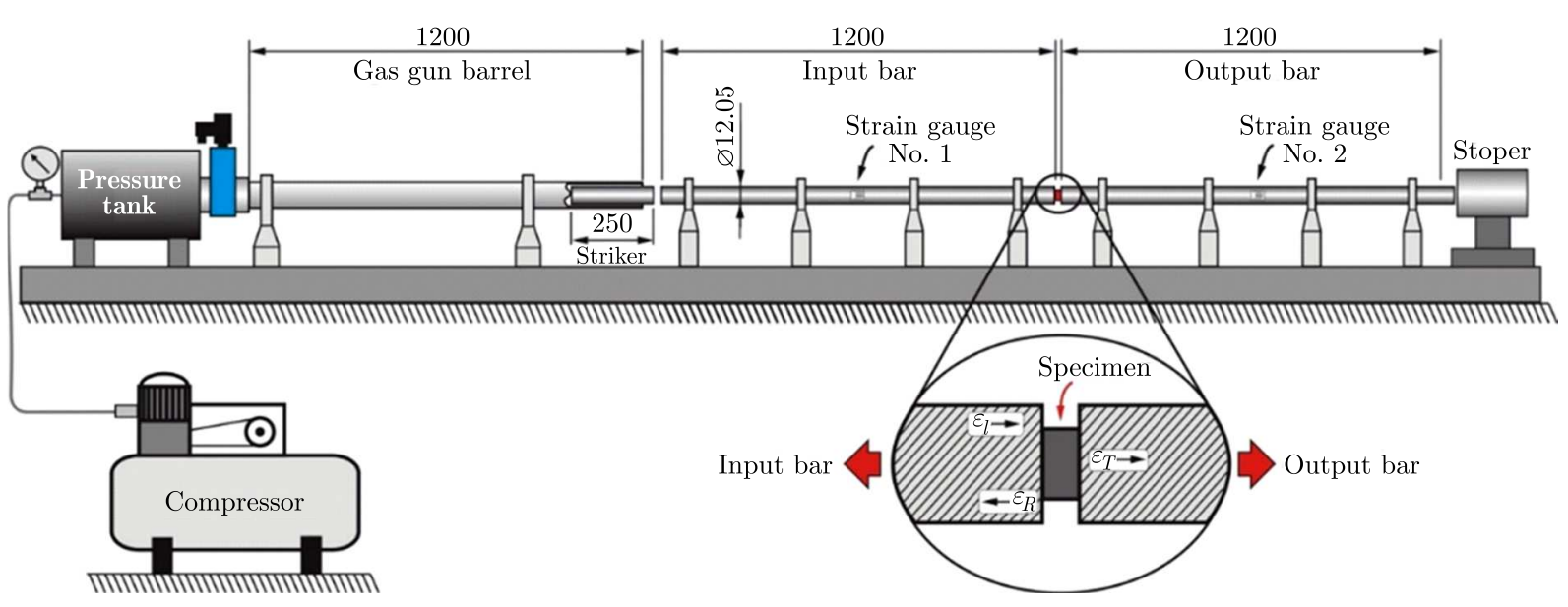

Fig. 2. Schematic diagram of the compression SHPB set-up (recording system not shown)

$C_{0}=4866 \mathrm{~m} / \mathrm{s}$ ). Each bar is supported by four linear bearing stands which are mounted on an optical bench allowing precise alignment of the bars system.

The wave signals in the input and output bars are captured using a pair of strain gauges attached symmetrically to the opposite surfaces of the bars. The strain gauges are connected to the opposite legs of the Wheatstone bridge, which is a typical half-bridge configuration. In the other legs of the bridge, dummy resistors are mounted, the resistance of which matches the strain gauges resistance $(350 \Omega)$. The foil strain gauges of $1.6 \mathrm{~mm}$ gauge length are used. The amplified signals of the strain gauges are recorded with a high cut-off frequency of $1 \mathrm{MHz}$ with the use of a signal conditioning unit and a high-speed digital oscilloscope.

\subsection{Numerical modelling}

It results from the principle of SHPB operation that a phenomenon occurring at a high strain rate is considered. In such cases, explicit time integration schemes are used in numerical analysis. In most cases, due to accuracy of the scheme, a central difference time integration scheme is used. A local truncation error of this method is of the order of $\Delta t^{2}$.

Such schemes are conditionally stable. The stability criteria are defined by the Courant-Friedrichs-Levy (CFL) principle (Courant et al., 1967):

$$
\Delta t \leqslant C_{C F L} \frac{\Delta x}{\sqrt{v_{a d v}^{2}+c_{\text {group }}^{2}}}
$$

where $\Delta t$ is the time step, $\Delta x$ - length interval, $C_{C F L}$ - Courant or CFL number, $v_{a d v}$ - advection velocity, $c_{\text {group }}$ - group velocity. In the case of typical analyses $C_{C F L}=0.9$. For analysis of phenomena in which detonation of explosives is considered, the blast wave interaction with the structure is equal to 0.67 . Whereas, in the case of numerical modeling of the phenomena taking place at very high strain rates, such as formation of an explosively formed projectile or shape charge jet generation, the adopted coefficient is even smaller.

The authors used the Finite Element Method (FEM) with a central difference time integration scheme implemented in explicit LS-Dyna to carry out numerical simulations (Hallquist, 2006).

The finite element model of the SHPB contains all components of the arrangement (Fig. 3). In addition to the main part of the set-up (bars, sample, striker), the model includes: pulse shaper, slide bearings and barrel. All parts, except for the sample and the pulse shaper, are described by eight-node solid elements with one point integration. Whereas, fully integrated solid elements with formulation for elements with a poor aspect ratio and accurate formulation 
are used to describe the sample and the pulse shaper (Hallquist, 2006). In order to reduce the number of finite elements and shorten computation time, symmetry of the model is utilized. In simulation, the dimensions of all elements of the SHPB are the same as in the experiments.

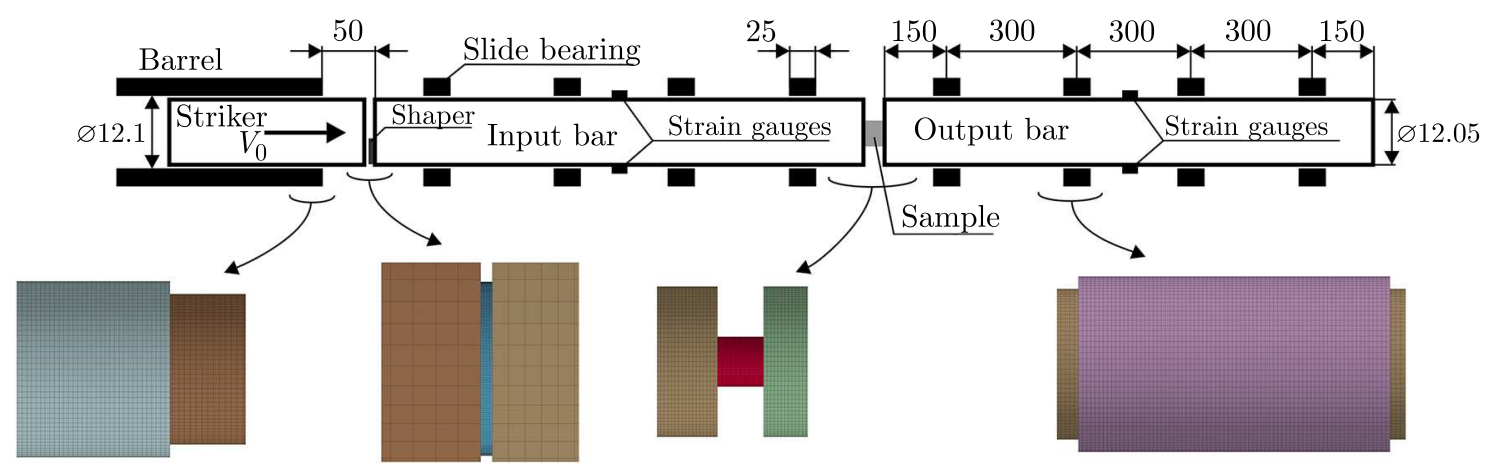

Fig. 3. A schematic diagram of the SHPB set-up used in numerical analysis

Between the interacting surfaces, there is defined contact based on a contact-impact algorithm the parameters of which have been established on the basis of the authors' own previous works (Panowicz, 2013). A segment-to-segment method (a mortar method) has been used to describe contact between the surfaces. This approach is based on the projection of integration points onto the master segment with penalty regularization of contact tractions (Fischer and Wriggers, 2006; Konyukhov and Schweizerhof, 2013). For the mortar method, the contact constraints are fulfilled in a weak manner. It shows optimal convergence behaviour compared to node-to-segment methods. Additionally, the Coulomb law is used to predict friction between the interacting surfaces. The friction coefficient is equal to 0.1 , which corresponds to greased surfaces.

The bars, striker, barrel and slide bearings are given material properties of maraging steel: Young's modulus $E_{B}=196 \mathrm{GPa}$, Poisson's ratio $\nu_{B}=0.3$, and density $\rho_{B}=7800 \mathrm{~kg} / \mathrm{m}^{3}$.

In this paper, the Johnson-Cook constitutive model (Rule and Jones, 1998) is used in the following form

$$
\sigma_{\text {flow }}=\left(A+B \varepsilon_{p}^{n}\right)\left(1+C \ln \dot{\varepsilon}^{*}\right)\left(1-T^{* m}\right) \quad T^{*}=\frac{T-T_{r}}{T_{m}-T_{r}}
$$

where $\dot{\varepsilon}^{*}=\dot{\varepsilon} / \dot{\varepsilon}_{0}$ is the normalized strain rate, $T^{*}$ is the homologous temperature and other symbols mean: $T$ - specimen temperature, $T_{m}$ - melting temperature of the specimen, $T_{r}-$ room temperature, $\dot{\varepsilon}$ - strain rate, $\dot{\varepsilon}_{0}$ - referenced strain rate usually equal to $1 \mathrm{~s}^{-1}, \varepsilon_{p}$ - plastic strain, and five material constants $A, B, n, C, m$ are applied to the sample and the pulse shaper. The first bracket in the Johnson-Cook constitutive equation describes strain hardening, the second - strain rate hardening, and the third one - thermal softening.

The constitutive relation is complemented by the hydrodynamic equation of state in the Gruneisen form (Steinberg, 1996; Hallquist, 2006)

$$
p= \begin{cases}\frac{\rho_{0} C_{0}^{2} \mu\left[1+\left(1-\frac{\gamma_{0}}{2}\right) \mu-\frac{a}{2 \mu^{2}}\right]}{1-\left(S_{1}-1\right) \mu-\frac{S_{2} \mu^{2}}{\mu+1}-\frac{S_{3} \mu^{3}}{(\mu+1)^{2}}}+\left(\gamma_{0}+a \mu\right) E & \text { for } \quad \mu \geqslant 0 \\ \rho_{0} C_{0}^{2} \mu+\left(\gamma_{0}+a \mu\right) E & \text { for } \quad \mu<0\end{cases}
$$

where $\mu=\rho / \rho_{0}-1, \rho$ is density, $\rho_{0}$ - initial density, $C_{0}$ - bulk sound speed, $\gamma_{0}$ - initial value of Gruneisen gamma, $a$ - coefficient of the volume dependence of gamma, $S_{1}, S_{2}, S_{3}$ - Hugoniot coefficients, $E$ - energy per volume. 
The material constants determining the behaviour of the semi-hard copper pulse shaper and the sample made of Ti-6Al-4V heve been taken from literature (Ozel and Sima, 2010; Grazka and Janiszewski, 2012) and are shown in Tables 1 and 2.

Table 1. Material constants of copper and Ti-6Al-4V (Ozel and Sima, 2010; Grazka and Janiszewski, 2012)

\begin{tabular}{|c|c|c|}
\hline $\begin{array}{c}\text { Physical } \\
\text { parameters }\end{array}$ & $\mathrm{Cu}$ & Ti-6Al-4V \\
\hline$\rho\left[\mathrm{kg} / \mathrm{m}^{3}\right]$ & 8940 & 4430 \\
\hline$E[\mathrm{GPa}]$ & 100 & 114 \\
\hline$\nu[-]$ & 0.31 & 0.3 \\
\hline$T_{m}[\mathrm{~K}]$ & 1338 & 1953 \\
\hline$c_{p}[\mathrm{~J} /(\mathrm{kg} \mathrm{K})]$ & 385 & 546 \\
\hline
\end{tabular}

\begin{tabular}{|c|c|c|}
\hline $\begin{array}{c}\text { J-C } \\
\text { constants }\end{array}$ & $\mathrm{Cu}$ & Ti-6Al-4V \\
\hline$A[\mathrm{MPa}]$ & 99.7 & 862.5 \\
\hline$B[\mathrm{MPa}]$ & 262.8 & 331.2 \\
\hline$n[-]$ & 0.23 & 0.34 \\
\hline$C[-]$ & 0.029 & 0.012 \\
\hline$m[-]$ & 0.98 & 0.8 \\
\hline
\end{tabular}

Table 2. EOS of copper and Ti-6Al-4V (Steinberg, 1996)

\begin{tabular}{|c|c|c|}
\hline & $\mathrm{Cu}$ & Ti-6Al-4V \\
\hline$C_{0}[\mathrm{~m} / \mathrm{s}]$ & 3940 & 5130 \\
\hline$S_{1}[-]$ & 1.489 & 1.028 \\
\hline$S_{2}[-]$ & 0.0 & 0 \\
\hline
\end{tabular}

\begin{tabular}{|c|c|c|}
\hline & $\mathrm{Cu}$ & Ti-6Al-4V \\
\hline$\gamma_{0}[-]$ & 2.02 & 1.23 \\
\hline$a[-]$ & 0.47 & 0.17 \\
\hline$S_{3}[-]$ & 0.0 & 0 \\
\hline
\end{tabular}

In the numerical analysis, the influence of the non-axisymmetric position of the pulse shaper as a function of its location, thickness and velocity of the striker is examined. The non-axisymmetric position of the pulse shaper is defined by the parameter $\delta$ which expresses the percentage offset of the shaper center relative to the longitudinal axis of the input bar

$$
\delta=\frac{h}{R-r} \cdot 100 \%
$$

where $R$ is the bar radius, $r$ - shaper radius equal to $1.5 \mathrm{~mm}, h$-distance between the shaper center and the symmetry axis of the bar.

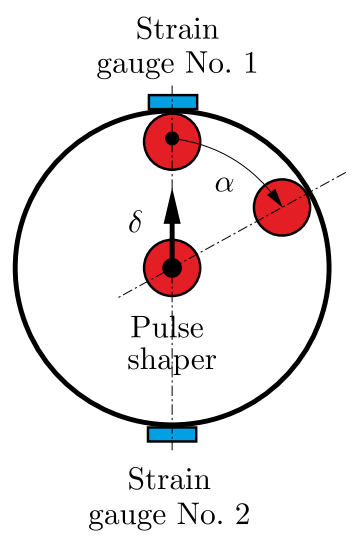

Fig. 4. Front surface of the incident bar from the striker side; $\delta$ - direction of the pulse shaper position change, $\alpha$ - angle between the gauge and the pulse shaper

Figure 4 shows schematically a change in the shaper position relative to the bar and strain gauges (parameter $\delta$ ). Considered are cases for the following values of the parameter $\delta: 0,20$, 40 and $60 \%$ for four shaper thicknesses $d=0.101,0.201,0.311,0.441$. The values of the shaper thickness are taken based on the measurements of thickness of the metal sheets the shaper is made of. Moreover, the above variants are examined in relation to different impact velocities $V_{0}$ of the striker, which are $10,12.5$ and $15 \mathrm{~m} / \mathrm{s}$. 
The validation process of the developed numerical model is based on a comparative analysis of the profiles of experimental and numerical incident waves. The validations have been performed for the experimental conditions shown in Table 3. The results of the experiments and their corresponding simulations collected in Figs. 5 and 6 show very good agreement.

Table 3. The experimental data

\begin{tabular}{|c|c|c|}
\hline $\begin{array}{c}\text { Shaper } \\
\text { thickness } \\
{[\mathrm{mm}]}\end{array}$ & $\begin{array}{c}\text { Shaper } \\
\text { location } \\
\delta[\%]\end{array}$ & $\begin{array}{c}\text { Shaper } \\
\text { velocity } \\
{[\mathrm{m} / \mathrm{s}]}\end{array}$ \\
\hline \hline 0.101 & 0 & 15.25 \\
\hline 0.101 & 100 & 15.03 \\
\hline 0.201 & 0 & 11.76 \\
\hline 0.201 & 100 & 12.62 \\
\hline 0.311 & 0 & 14.16 \\
\hline 0.311 & 100 & 15.06 \\
\hline 0.441 & 0 & 11.24 \\
\hline 0.441 & 100 & 14.6 \\
\hline
\end{tabular}

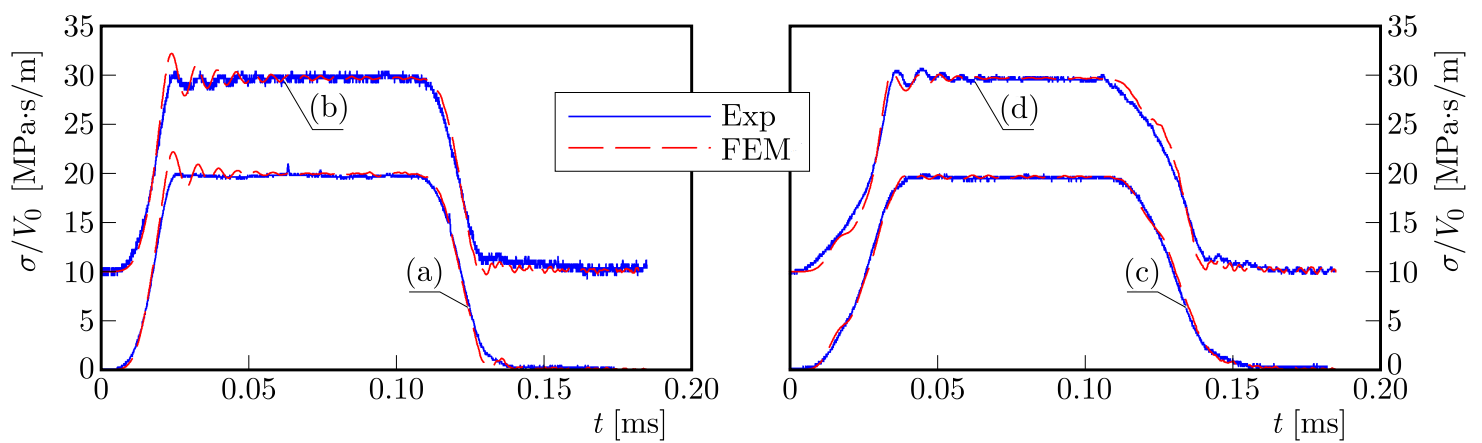

Fig. 5. Comparison of the normalized incident wave profiles obtained from numerical (FEM) and experimental (Exp.) analysis: (a) $d=0.101, \delta=0 \%$, (b) $d=0.101, \delta=100 \%$, (c) $d=0.201, \delta=0 \%$,

(d) $d=0.201, \delta=100 \%$, curves $b$ and $d$ are shifted upwards for better illustration of the results

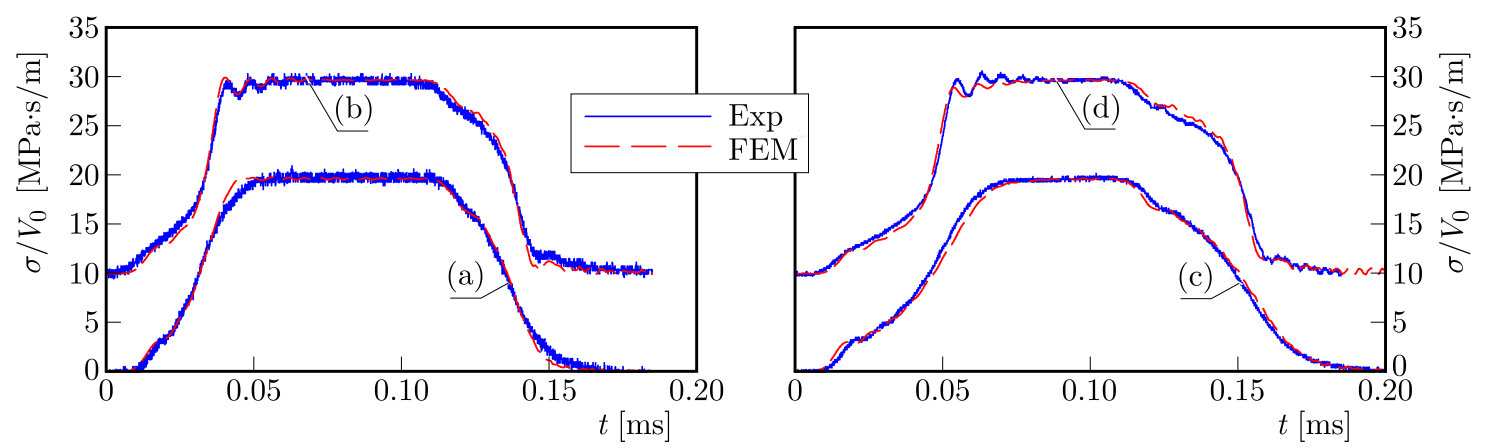

Fig. 6. Comparison of the normalized incident wave profiles obtained from numerical (FEM) and experimental (Exp.) analysis: (a) $d=0.311, \delta=0 \%$, (b) $d=0.311, \delta=100 \%$, (c) $d=0.441, \delta=0 \%$,

(d) $d=0.441, \delta=100 \%$, curves $b$ and $d$ are shifted upwards for better illustration of the results 


\section{Results and discussion}

The plain fact is that in the case of a half- or a full-Wheatstone bridge, a non-axisymmetric placement of the pulse shaper is not clearly reflected in the shape of the wave profiles recorded by strain gauges. In fact, it is a consequence of the compensation effect resulting from the position of the strain gauges on the opposite side surfaces of the bar. However, in the case of application of the Wheatstone bridge arrangement based on the quarter-bridge circuit (commonly used in SHPB experiments), disturbances resulting from the non-axisymmetric position of the pulse shaper become significant. Figure 7 presents summary profiles of the incident, reflected and transmitted waves depending on the relative position of the pulse shaper and strain gauges.

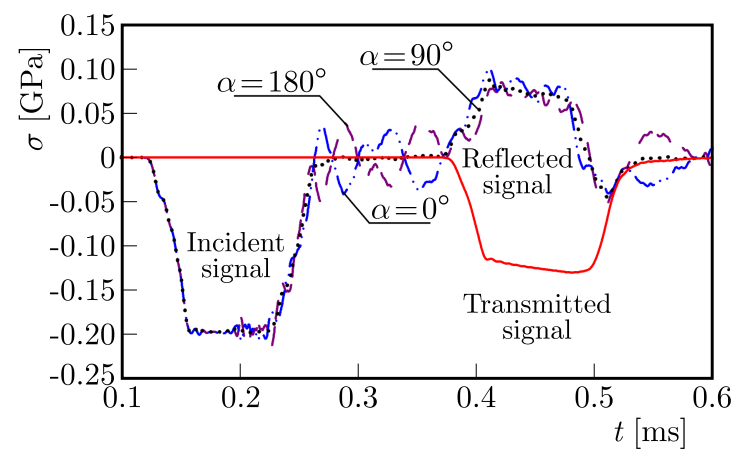

Fig. 7. Disturbances of waves signals depending on the relative position of the pulse shaper and strain gauges (parameter $\delta=60 \%, \alpha=0^{\circ}, 90^{\circ}$ and $180^{\circ}$, pulse shaper thickness $d=0.211 \mathrm{~mm}$,

impact velocity $V_{0}=10 \mathrm{~m} / \mathrm{s}$ )

As expected, with the increasing parameter $\delta$, the disturbance amplitudes of the incident and reflected signals are also increasing. However, only a part of the incident signal is disturbed, whereas the whole reflected signal profile is distorted by bending waves. In turn, when the non-axisymmetric position of the pulse shaper is not in the direction of the strain gauge but inclined at some angle $\alpha$, the recorded disturbance amplitudes of wave signals decrease with the increasing angle of deflection. The smallest oscillations of wave signals occurr when the deflection angle is equal to $90^{\circ}$ (green line).

On the basis of Fig. 7, it can also be seen that the transmitted signal is noise-free and does not depend on the non-axisymmetric position of the pulse shaper. Such a situation takes place and is preferable in the case of high-strain-rate material testing, when the material specimen is not permanently connected to the contact surfaces of the bars, for example, in uniaxial compression. However, when tensile or torsion tests are conducted, the transmitted signal will also be disturbed by the bending waves.

For a typical half-bridge configuration (strain gauges are connected to the opposite bridge legs), disturbances caused by the non-axisymmetric position of the pulse shaper have a different character and their amplitude is significantly smaller. This is due to cancellation of the bending effect (flexural mode of vibration) by averaging strain (Fig. 4). In Fig. 8, it can be observed that the incident wave shapes differ to a small extent for different values of the parameter $\delta$ (Figs. $8 \mathrm{~b}$ and $8 \mathrm{~d}$ ), whereas for the pulse shaper with thickness of 0.441 , the differences are larger compared to the same profiles obtained for the shaper with thickness of 0.201. Furthermore, characteristic/additional disturbances resembling concavity in the incident wave profiles for their plateau parts are observed (Fig. 8d). The greater concavity, the higher value of the parameter $\delta$. A similar effect of the non-axisymmetric pulse shaper position is observed for reflected waves (Fig. 9). However, the magnitudes of the observed disturbances of the reflected wave profile are smaller. 
(a)

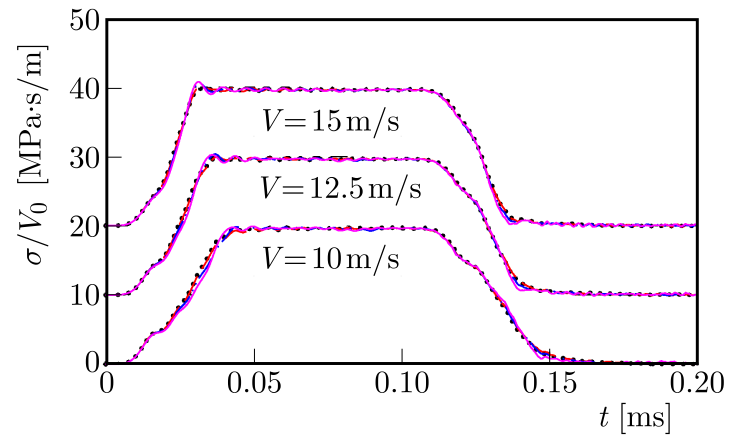

(c)

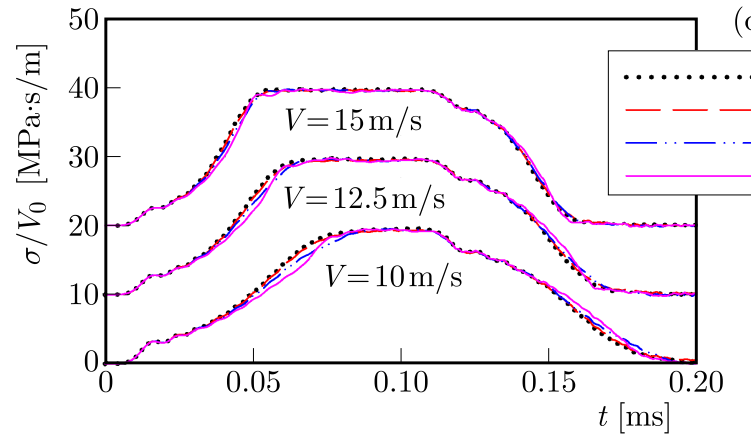

(b)

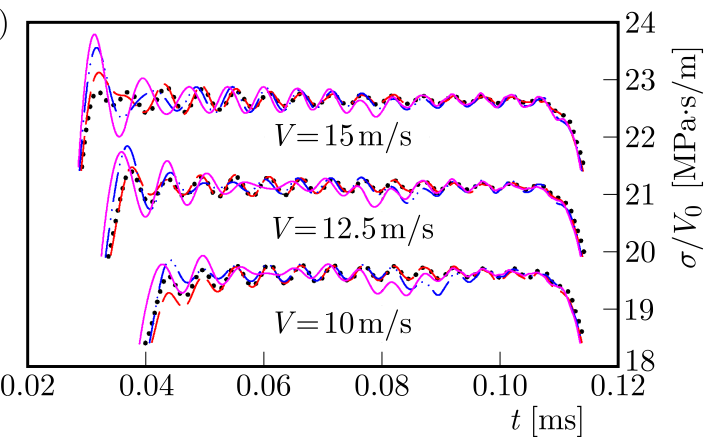

(d)

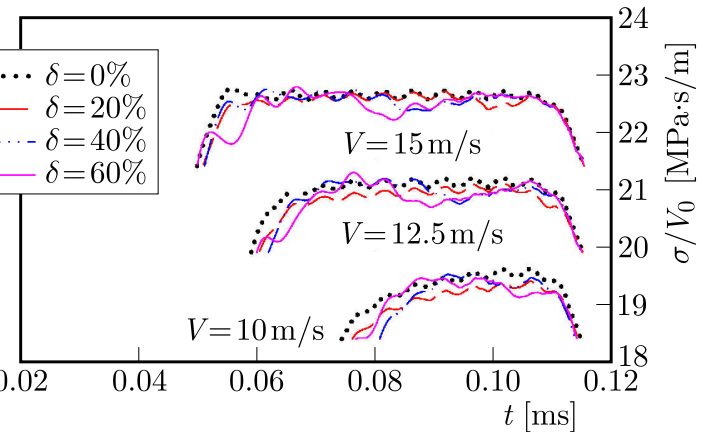

Fig. 8. The incident stress waves normalized to impact velocity: shaper thickness $d=0.201 \mathrm{~mm}$ (a), (b) and $d=0.441 \mathrm{~mm}(\mathrm{c}),(\mathrm{d})$, curves for $V=12.5 \mathrm{~m} / \mathrm{s}$ and $V=15 \mathrm{~m} / \mathrm{s}$ are shifted upwards for better illustration of the results

(a)

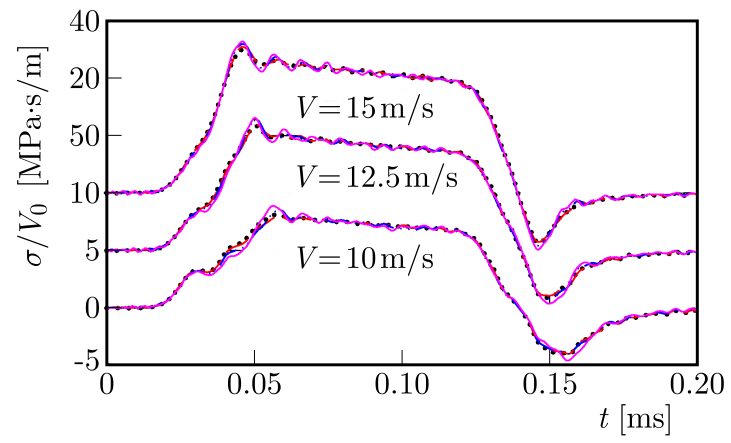

(b)

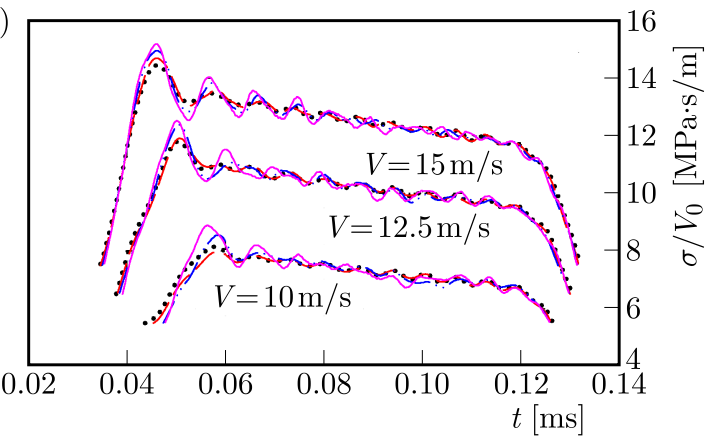

(c)

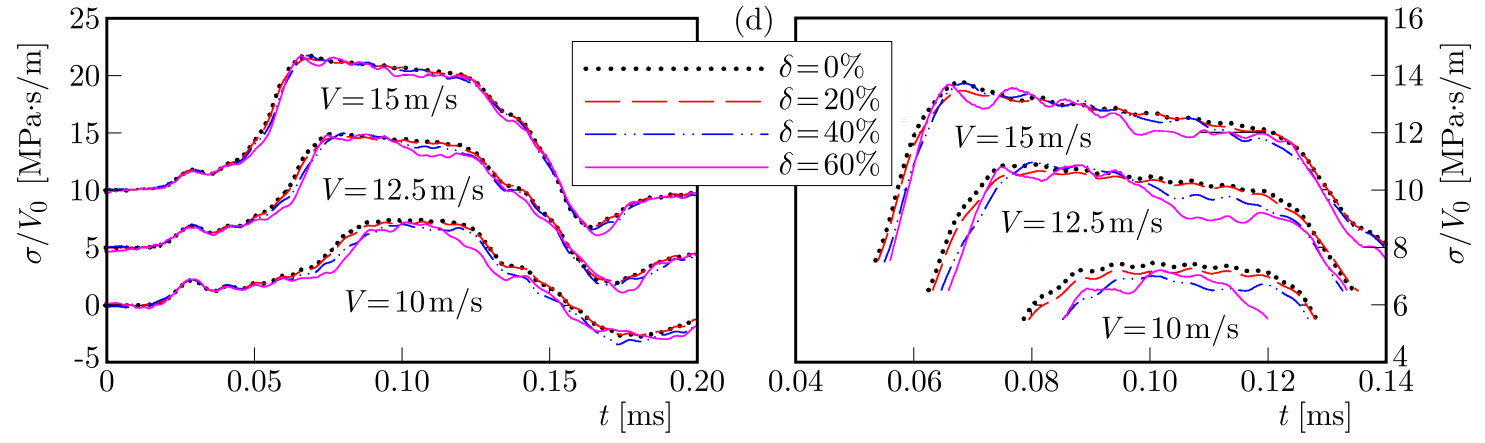

Fig. 9. The normalized reflected stress waves normalized to impact velocity: shaper

thickness $d=0.201 \mathrm{~mm}$ (a), (b) and $d=0.441 \mathrm{~mm}$ (c), (d), curves for $V=12.5 \mathrm{~m} / \mathrm{s}$ and $V=15 \mathrm{~m} / \mathrm{s}$ are shifted upwards for better illustration of the results

For small pulse shaper thickness, the influence of the non-axisymmetric position of the pulse shaper is manifested in the incident waves, particularly, by larger initial amplitudes of the Pochhammer and Chree disturbances (Fig. 10). This effect decreases with the increasing velocity of the striker and an increase in the pulse shaper thickness. If a thicker pulse shaper is used, 
the disturbance occurs in the form of concavity in the other half of the signal plateau. It is found that the concavity magnitude is less dependent on the striker velocity. Moreover, the non-axisymmetric position of the pulse shaper affects the rise time of the incident pulse especially for lower striker velocity and the thicker pulse shaper. Similar relations are also observed for the reflected signals.

(a)

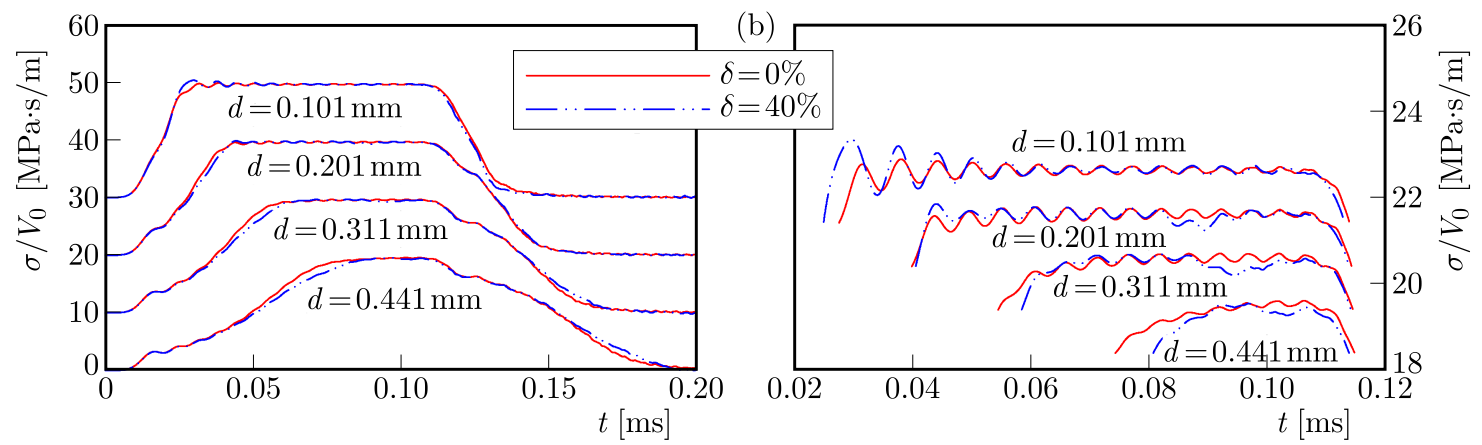

(c)

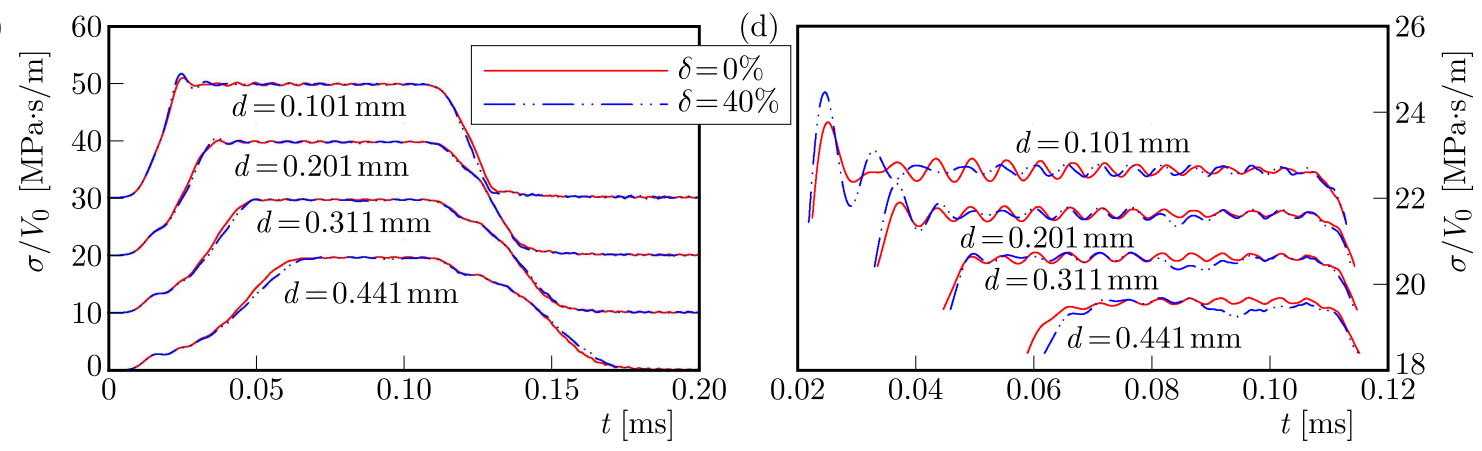

(e)

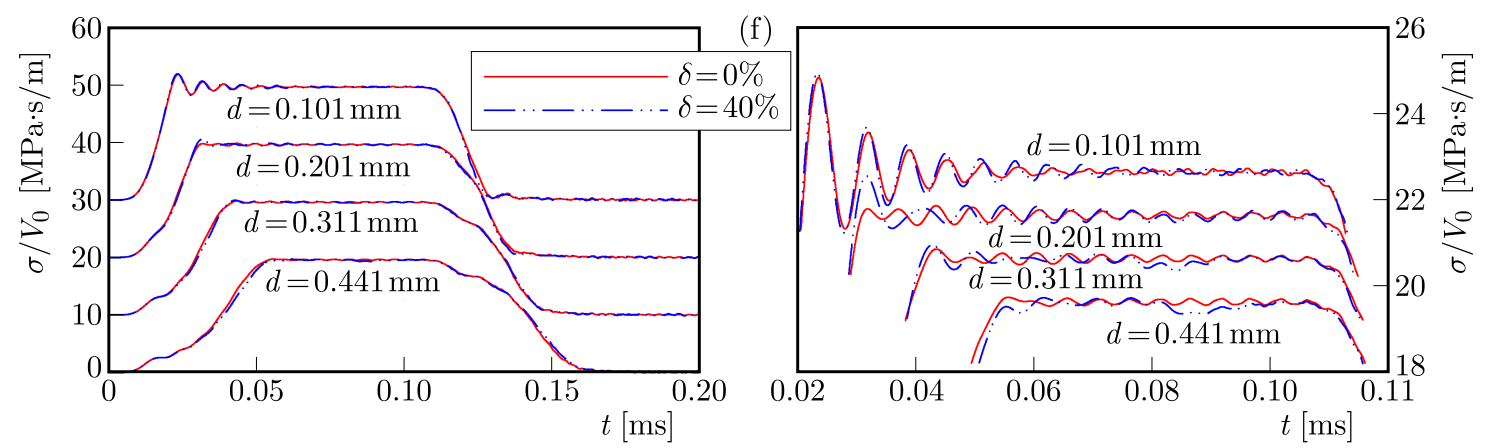

Fig. 10. Incident stress waves for $\delta=0 \%$ and $40 \%$ and different shaper thickness: (a), (b) $V_{0}=10 \mathrm{~m} / \mathrm{s}$, (c), (d) $V_{0}=12.5 \mathrm{~m} / \mathrm{s}$, (e), (f) $V_{0}=15 \mathrm{~m} / \mathrm{s}$, curves for $d$ from 0.101 to $0.311 \mathrm{~mm}$ are shifted upwards for better illustration of the results

In order to fully assess the impact of the non-axisymmetric position of the pulse shaper, the $\sigma-\varepsilon$ curves for Ti-6Al-4V have been determined according to Kolsky's theory using 3-wave analysis

$$
\varepsilon=\frac{C_{B}}{L_{S}} \int_{0}^{t}\left(\varepsilon_{I}-\varepsilon_{R}-\varepsilon_{T}\right) d \tau \quad \sigma=\frac{1}{2} \frac{A_{B}}{A_{S}} E_{B}\left(\varepsilon_{I}+\varepsilon_{R}+\varepsilon_{T}\right)
$$

where $E_{B}, C_{B}$ - longitudinal elastic wave speed and Young's modulus of the bar material, $L_{S}-$ initial length of the specimen, $A_{B}, A_{S}$ - cross-sectional areas of the bars and the specimen, respectively; and of the bar material, $\varepsilon_{I}, \varepsilon_{R}$ and $\varepsilon_{T}-$ incident, reflected and transmitted strain, respectively. 
Figures 11 and 12 show the influence of the non-axisymmetric position of the pulse shaper expressed with the parameter $\delta$ and thickness $d$ of the pulse shaper as a function of the striker velocity on the $\sigma-\varepsilon$ curve profile. These figures illustrate that the non-axisymmetric position of the pulse shaper generates a deviation in flow stress and the final strain values from the perfect condition $(\delta=0)$ in the initial loading. In the case of a thin pulse shaper $(d=0.101$ and $0.201 \mathrm{~mm})$, the deviations are smaller than for shaper thickness of 0.311 and $0.441 \mathrm{~mm}$ (Fig. 12). Visible

(a)

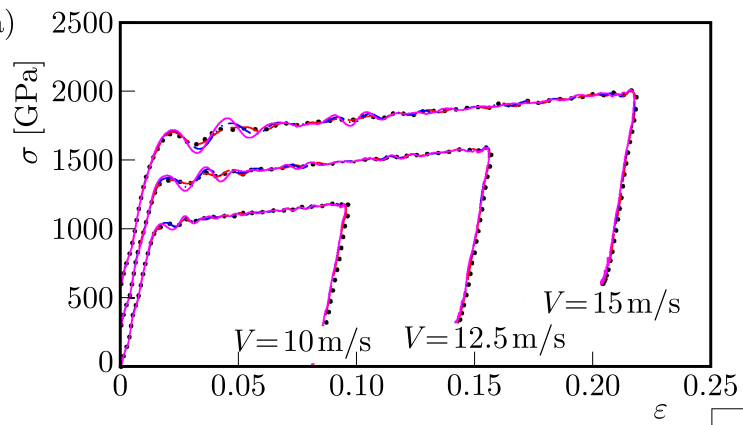

(b)

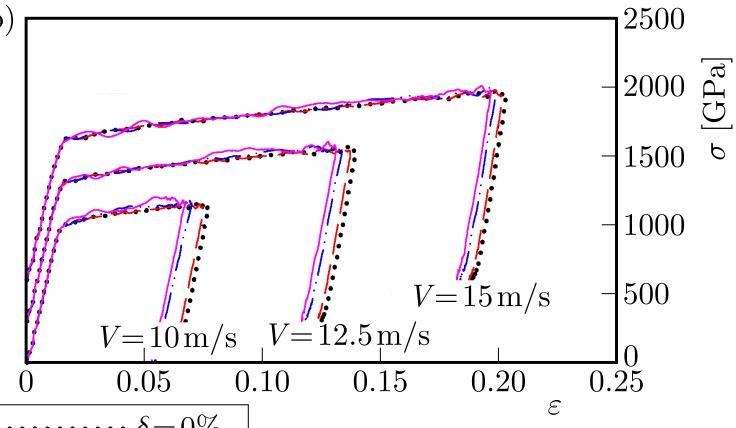

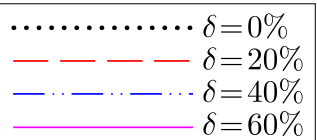

Fig. 11. The comparison of stress-strain curves for different parameters $\delta$ and pulse shaper thickness: (a) $d=0.201 \mathrm{~mm}$, (b) $d=0.441 \mathrm{~mm}$, curves for $d$ from 0.101 to $0.311 \mathrm{~mm}$ are shifted for better illustration of the results

(a)

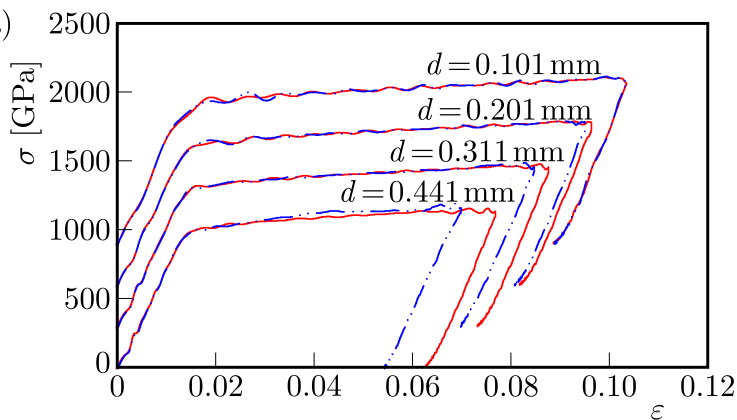

(c)

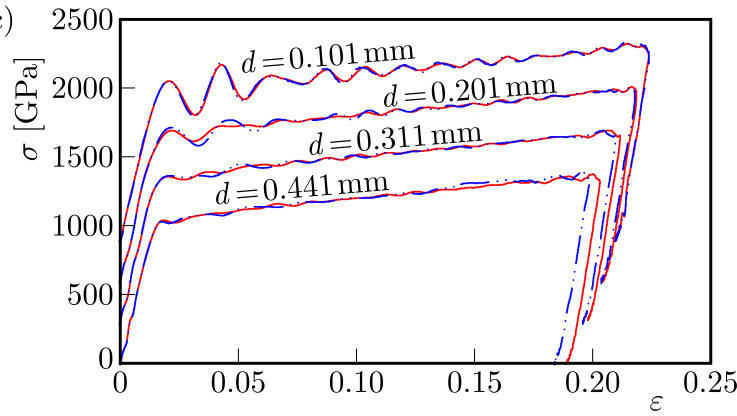

(b)
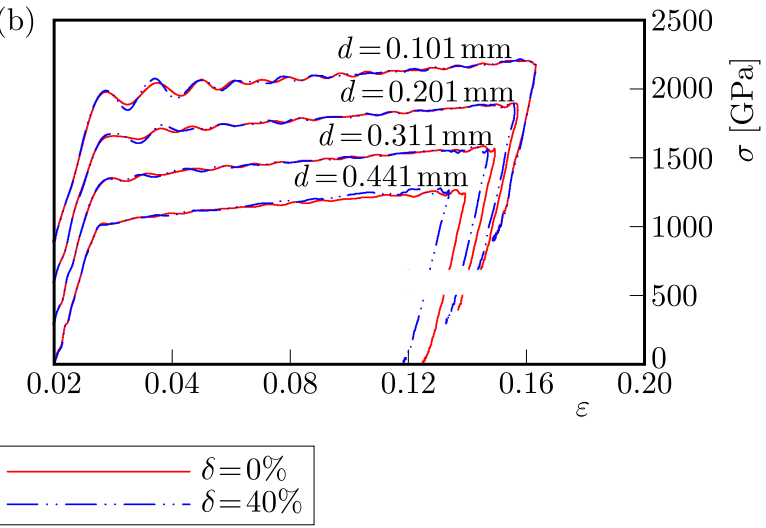

Fig. 12. The comparison of stress-strain curves for parameters $\delta=0$ and $40 \%$ : (a) $V_{0}=10 \mathrm{~m} / \mathrm{s}$, (b) $V_{0}=12.5 \mathrm{~m} / \mathrm{s}$, (c) $V_{0}=15 \mathrm{~m} / \mathrm{s}$, curves for $d$ from 0.101 to $0.311 \mathrm{~mm}$ are shifted upwards for better illustration of the results

deviations in Fig. 11b constitute a fragment of the plateau portion of the curve $\sigma-\varepsilon$ (flow stresses at the latter period) and a curve part showing the final strain values. It should be noted that for the lowest velocity of the striker $\left(V_{0}=10 \mathrm{~m} / \mathrm{s}\right)$, the deviations are significantly higher than for higher striker velocity $\left(V_{0}=12.5 \mathrm{~m} / \mathrm{s}\right.$ and $\left.15 \mathrm{~m} / \mathrm{s}\right)$, and it can be supposed that with the increasing impact velocity, the deviation will decrease. Maximum deviations for $\delta=60 \%$ in the $\sigma-\varepsilon$ curve plateau part are $57,53,21 \mathrm{MPa}$ for the striker velocity of $10,12.5$ and $15 \mathrm{~m} / \mathrm{s}$, respec- 
tively, and the final strain values for $\sigma=800 \mathrm{MPa}$ level are 0.0092, 0.0086, 0.0066, respectively. In the case of the parameter $\delta=20 \%$, such disturbances are very small and can be neglected. It is also possible to neglect the impact of the aforementioned parameters on the flow stresses in the elastic regime. No disturbances in this area can be explained by the fact that the flexural wave velocity is relatively small compared to the longitudinal wave velocity and interferes only with the waves at the later period, producing highly distorted data. In turn, the deviation in the final strain is due to some portions of the impact energy being dissipated as flexural energy.

\section{Conclusion}

The influence of the non-axisymmetric pulse shaper position in the split Hopkinson pressure bar technique has been investigated numerically. Our studies show that the distorted signal of the SHPB is mainly due to the presence of flexural modes of vibration, like in the misalignment bars effect (Kariem et al., 2012). The results of analysis of a wide range of numerical experiments indicate that the effect of the non-axisymmetric pulse shaper position may be neglected if deviation from the input bar axis does not exceed $20 \%$, which corresponds to the position deviation (distance between the shaper centre and symmetry bar axis) equal to approximately $15 \%$ of the input bar radius.

It should be emphasised that the above discussion has shown only one configuration of the bars supports position, i.e., the number of supports and the distance between them. In the case of another configuration, especially in the case of a smaller number of the bars supports, the non-axisymmetric pulse shaper position will produce a different deviation of SHPB data. A similar situation will take place when only one strain gauge configuration in the SHPB arrangement is considered. In that case, the deviation amplitude of the measured signals will be larger and, in consequence, bigger errors will appear in the SHPB data (stress-strain curve).

\section{Acknowledgement}

The paper has been partially founded within the project DOBR-BIO4/031/13249/2013 supported by the National Centre for Research and Development. This support is gratefully acknowledged.

\section{References}

1. Bekker A., Cloete T.J., Chinsamy-Turan A., Nurick G.N., Kok S., 2015, Constant strain rate compression of bovine cortical bone on the split-Hopkinson pressure bar, Materials Science and Engineering $C$, 46, 1, 443-449

2. Chen W., Luo H., 2004, Dynamic compressive responses of intact and damaged ceramics from a single split Hopkinson pressure bar experiment, Experimental Mechanics, 44, 3, 295-299

3. Chen W., Song B., 2011, Split Hopkinson (Kolsky) Bar: Design, Testing and Applications, Berlin, Springer

4. Chen W., Song B., Frew D.J., Forrestal M.J., 2003, Dynamic small strain measurements of a metal specimen with a split Hopkinson pressure bar, Experimental Mechanics, 43, 1, 201-23

5. Cloete T.J., van der Westhuizen A., Kok S., Nurick G.N., 2009, A tapered striker pulse shaping technique for uniform strain rate dynamic compression of bovine bone, EDP Sciences, $\mathbf{1}$, 901-907

6. Courant R., Friedrichs K.O., Lewy H., 1967, Ueber die partiellen Differenzengleichungen der mathematischen Physik, Mathematische Annalen (1928), 100, 32-74. Translated as: On the partial difference equations of mathematical physics, IBM Journal of Research and Development, $11,215-234$ 
7. Ellwood S., Griffiths L.J., PArry D.J., 1982, Materials testing at high constant strain rates, Journal of Physics, E, 15, 280-282

8. Fischer K.A., Wriggers P., 2006, Mortar based frictional contact formulation for higher order interpolations using the moving friction cone, Computer Methods in Applied Mechanics and Engineering, 195, 5020-5036

9. Foley J.R., Dodson J.C., McKinion C.M., 2010, Split Hopkinson bar experiments of preloaded interfaces, Proceedings of the IMPLAST 2010 Conference

10. Follansbee P.S., 1985, The Hopkinson Bar in Mechanical Testing and Evaluations, ASM Handbook, 9th ed. ASM Int., Materials Park Ohio

11. Frantz C.E., Follansbee P.S., 1984, Experimental techniques with the split Hopkinson pressure bar, Proceedings of the 8th International Conference on High Energy Rate Fabrication, San Antonio, Texas, 229-236

12. Frew D.J., Forrestal M.J., Chen W., 2002, Pulse shaping techniques for testing brittle materials with a split Hopkinson pressure bar, Experimental Mechanics, 42, 1, 93-106

13. Frew D.J., Forrestal M.J., Chen W., 2005, Pulse shaping techniques for testing elastic-plastic materials with a split Hopkinson pressure bar, Experimental Mechanics, 45, 186-195

14. Grazka M., Janiszewski J., 2012, Identification of Johnson-Cook equation constants using finite element method, Engineering Transactions, 60, 215-223

15. Hallquist J.O., 2006, Ls-Dyna. Theoretical Manual, California, Livermore Software Technology Corporation

16. Hsiao H.M., Daniel I.M., 1998, Dynamic compressive behavior of thick composite materials, Experimental Mechanics, 38, 172-180

17. Kariem M.A., Beynon J.H., Ruan D., 2012, Misalignment effect in the split Hopkinson pressure bar technique, International Journal of Impact Engineering, 47, 60-70

18. Kolsky H., 1949, An investigation of the mechanical properties of materials at very high rates of strain, Proceedings of the Physical Society Section B, 62, 11, 676-700

19. Konyukhov A., Schweizerhof K., 2013, Computational Contact Mechanics, Geometrically Exact Theory for Arbitrary Shaped Bodies, Berlin, Springer

20. Li W., XUA J., 2009, Impact characterization of basalt fiber reinforced geopolymeric concrete using a 100 mm-diameter split Hopkinson pressure bar, Materials Science and Engineering A, 513-514, $145-153$

21. LU Y.B., Li Q.M., 2010, Appraisal of pulse-shaping technique in split Hopkinson pressure bar tests for brittle materials, International Journal of Protective Structures, 1, 3, 363-390

22. Naghdabadi R., Ashrafia M. J., Arghavanic J., 2012, Experimental and numerical investigation of pulse-shaped split Hopkinson pressure bar test, Materials Science and Engineering A, 539, 285-293

23. Naik N. K., Yernamma P., 2008, Mechanical behaviour of acrylic under high strain rate tensile loading, Polymer Testing, 27, 504-512

24. Nemat-Nasser S., Isaacs J.B., Starrett J.E., 1991, Hopkinson techniques for dynamic recovery experiments, Proceedings of the Royal Society, 435, 371-391

25. Ozel T., Sima M., 2010, Finite element simulation of high speed machining Ti-6Al-4V alloy using modified material models, Transactions of NAMRI/SME, 38, 49-56

26. Panowicz R., 2013, Analysis of selected contact algorithms types in terms of their parameters selection, Journal of KONES Powertrain and Transport, 20, 1, 263-268

27. Ramirez H., Rubio-Gonzalez C., 2006, Finite-element simulation of wave propagation and dispersion in Hopkinson bar test, Materials and Design, 27, 36-44 
28. Rule W.K., Jones S.E., 1998, A revised form for the Johnson-Cook strength model, International Journal of Impact Engineering, 21, 8, 609-624

29. Shemirani A. B., Naghdabadi R., Ashrafi M.J., 2016, Experimental and numerical study on choosing proper pulse shapers for testing concrete specimens by split Hopkinson pressure bar apparatus, Construction and Building Materials, 125, 326-336

30. Song B., Chen W., 2004, Loading and unloading split Hopkinson pressure bar pulse-shaping techniques for dynamic hysteretic loops, Experimental Mechanics, 44, 6, 622-627

31. Song B., Chen W., 2005, Split Hopkinson pressure bar techniques for characterizing soft materials, Latin American Journal of Solids and Structures, 2, 113-152

32. SteinberG D.J., 1996, Equation of state and strength properties of selected materials, LLNL Report, UCRL-MA-106439

33. VECCHIO K.S., JiAng F., 2007, Improved pulse shaping to achieve constant strain rate and stress equilibrium in split-Hopkinson pressure bar testing, Metallurgical and Materials Transactions A, 38, 2655-2665 